\title{
The endoscopic treatment of gastrointestinal symptoms
}

\author{
P. SPINELLI*
}

\section{Introduction}

Nearly all the digestive cancers are endocavitary and thus endoscopy is the most suitable approach for them, for diagnostic as well as for therapeutic purposes. Digestive cancers form about 20 percent of all diagnosed cancers; when these are advanced, most of them are poorly responsive to curative treatments; consequently, patients not responding to curative treatment will need symptomatic, palliative treatment.

Flexible endoscopy continuously improves its therapeutic possibilities and is used to treat a variety of diseases traditionally managed by surgery. Palliation is the treatment of the symptoms of a disease. Palliative treatment is planned when it is impossible to treat a disease for cure. Palliation would be better defined by dividing it into palliative care, including the treatments required to bridge the patients with advanced tumors from a specific disease status to the terminal events, and control of symptoms, concerning a former phase in the natural history of the disease, when an acceptable disease-related quality of life is obtainable.

In view of these distinctions, palliative treatments to control symptoms should start as soon as the disease is classified as being incurable. It could happen at the time of the diagnosis, if conditions preventing curative treatments already exist. Palliation must be undertaken if anticancer treatments are not advisable because of general or local reasons; in such cases, a therapeutic approach directed to treat the cancer would waste time, opportunities and biological resources that could be used for a more tolerable and profitable symptomatic approach.

Diagnosis of a solid cancer must be followed by the staging, as therapeutic options and prognosis are strictly related to the stage. Staging procedures are based on sophisticated and precise diagnostic tools, so that the oncologist should be able to separate localized from diffused and curable from non-curable diseases in the majority of cases. In fact, the suitable diagnostic possibilities must be implemented, to single out non-curable patients as early as possible, avoiding to give them inefficacious, sometime toxic and always costly anticancer treatments, and managing them in order to the symptoms they are complaining.

Palliative option has to be undertaken only after having considered the different curative potentialities of surgery and radio-, chemo-, immunotherapy, and of any other kind of certified treatments, as well as the risk conditions of a particular patient, the side effects of a treatment, the quality of the remaining life, and weigh up the real impact of the therapeutic procedures with regard to the expected benefits.

Analysis of gene expression patterns may be useful in the future for predicting the response to an anticancer treatment. The availability of new prognostic indicators in the form of molecular, clinical, and pathologic testing might offer the possibility of selecting potentially curable from non-curable patients.

* Direttore U.O.C. Diagnostica e Chirurgia Endoscopica

Istituto Nazionale Tumori di Milano 


\section{Palliation milestones}

\section{Surgery}

Surgery is generally the first palliative option. From the surgeon's standpoint, therapy is considered palliative when resection of all known tumor sites is no longer possible or advisable. When a cure is not possible, the goal of treatment and eventually the success of therapy becomes judged by the control of symptoms and alleviation of suffering. The process of providing palliative care may be a departure from the traditional surgical satisfaction derived from the complete excision of a malignancy, but surgeons achieving excellence in palliative care will likely find this a rewarding endeavor.

A part of the gastrointestinal tract may be resected in presence of painful obstructive symptoms with the aim of relieving pain, restoring the lumen and reducing bleeding by removing a tumor. Bypass operations, indicated in cases of non-resectable tumor masses, may be performed through traditional laparotomy and also through laparoscopic access, achieving the double goal of minimum trauma and quick recovery. The appropriate use of surgery in these settings can improve the quality of life of patients with cancer.

\section{Radiotherapy}

Radiotherapy reduces the volume of unresectable tumors. Large cancers may be reduced and become resectable. Under specific circumstances, radiotherapy can be given together with endoscopic treatments, thus combining endoluminal with the extraluminal benefits of mass reduction. Among the different radiotherapeutic options, brachytherapy can better localize radiation dose with limited side effects; this is important when treating previously irradiated areas. In some cancers causing local symptoms, reduction of the size and of the extravisceral extension of the tumor with radiation therapy results in partial control of pain.

\section{Chemotherapy}

Chemotherapy for palliation of advanced unresectable or metastatic cancer is used to reduce the size of tumor masses and to alleviate symptoms. Furthermore, in patients who have locally advanced, unresectable disease and in patients in whom tumors are resected with positive margins the duration of survival can be increased with palliative chemotherapy and irradiation. Along with chemotherapy, endoscopic treatments aimed at immediately relieving obstruction of an occluded cavity may be strongly advisable in selected cases, allowing for the functional recovery.

\section{Palliation of gastrointestinal symptoms}

Related to their location, primary digestive cancers as well as their metastases can compromise esophageal, tracheobronchial, biliary, and urinary functions; consequently, palliative care should not be limited to the patients with preterminal disease, but greatly expanded, starting with the control of symptoms as soon as the disease is classified as incurable and avoiding anticancer treatments when unnecessary.

In recent years the continuing increase of the lifespan entails a constant increase in the incidence of age-related malignant neoplasms; advanced age, together with related risk factors and concurrent diseases, reduce the possibility of performing radical treatments and opens the doors to palliative treatments. In current clinical practice, however, most patients are treated with curative intent, even when palliation of symptoms would have been the right choice. Furthermore, the majority of clinical trials currently in progress are evaluating the response to treatments in terms of decrease in the volume of the tumor mass and global survival, but neglecting the evaluation of the impact of the treatment toxicity and of the general side effects on the quality of life and on the relationships of the patients with the people around them.

The palliative treatment of the tumors of the esophagus, trachea, or intestinal, biliary, or urinary tracts, very often results in an effective extension of the survival time and improves the performance status, although these are not the primary purposes of a palliative procedure. 
Consequently palliation becomes, in many cases, not just the simple treatment of symptoms, but it offers to the patient a wide range of therapeutic opportunities during the entire course of the disease.

Endoscopic palliative treatments aim to obtain the best possible quality of life with immediate and durable benefits with negligible trauma, side effects and incidence of complications related to the proposed advantages. Although these objectives seem to be obvious, it often happens that these simple principles - essential to the correct approach to the oncologic patient - are not adopted and patients are submitted to treatments that are not suited to their requirements and their health status. In everyday practice it frequently happens that patients who only need control of symptoms related to the size and site of the tumor masses and to their relations with the surrounding anatomical structures are overtreated.

Under these conditions, a palliative treatment should be planned in agreement with the patient and possibly tailored on the patient's willings, so that the quality of life offered by the treatment could be consistent with the patient's lifestyle. This is because there are different ways to achieve relief from one symptom. The physician must be able to inform the patients about the different methods of palliation available so that they can choose the one that fits better with their preferred lifestyle. For example, esophageal stenosis can be relieved by a nasogastric tube, by a laser treatment, by a gastrostomy, by a stent insertion or by a palliative radiochemotherapy: patients must be informed about these options so that they can decide which one is the most suitable for their way of life.

Gastrointestinal symptoms may be produced by digestive or extradigestive tumors. Most of these tumors affect the digestive cavities and grow into them, occupying the spaces required for the digestive functions. The gastrointestinal tract has cavities that function as 'containers' or as 'canals' (stomach, esophagus, intestine, biliary and pancreatic tract). Tumors reduce the space available and impair the functions of containing and flowing; moreover, tumors that infiltrate and ulcerate the walls of these cavities generate symptoms. In particular, they cause hemorrhage, obstruction, perforation, and fistula formation. The most important symptoms of digestive tumors are: dysphagia, regurgitation, salivation, vomiting, jaundice, pain, and hemorrhage. All these symptoms can be treated by endoscopic modalities.

\section{Dysphagia}

Malignant dysphagia can be in relation to the presence of a primary or secondary esophageal tumor or it can be consequent to a surgical treatment or to a radiotherapy or chemotherapy. Dysphagia is the initial symptom of an esophageal cancer in 90 percent of cases, but it also may be caused by compression or infiltration by thyroid, lung or pleural tumors, mediastinal lymphomas or by metastatic involvement of the mediastinum, mainly by breast cancer. Dysphagia can be associated with pain (odynophagia) and aspiration of food and saliva into the trachea and bronchi and with chronic cough, asthma, laryngitis, and, eventually, pneumonia. Beyond the most common causes, in oncologic patients dysphagia may be due to:

- neurologic reasons, e.g., cricopharyngeal dysphagia because of recurrent nerve palsy due to perineural and neural infiltration by tumor tissue; neurologic dysphagia may be also due to vagal or sympathetic tumoral infiltration, with involvement of the skull base or due to brain metastases;

- mucositis related to candidosis, bacterial infection, herpes, radiotherapy, chemotherapy;

- asthenia/cachexia.

Esophagoscopy is indicated when a patient complains of dysphagia. It allows the surgeon to characterize and exactly locate a tumor. Echoendoscopy is extremely useful for determining the involvement of neighboring anatomical structures. Infiltration of the wall interrupts the progression of peristaltic contraction and stops the progression of food.

\section{Regurgitation, salivation}

The regurgitation of undigested material is caused by esophageal stenoses with incompetence of the upper esophageal sphincter. It can induce inhalation (aspiration of this material into the airways). Total obstruction stops the passage of saliva and causes the onset of another invalidating symptom, that is salivation. 
The patient complaining of salivation is obliged to spit or dribble continuously and walks around with a bag full of handkerchiefs, deprived of a social life. Dysphagia can be associated with odynophagia, generally caused by inflammation of the esophageal wall or by candidiasis or herpesvirus; odynophagia too may cause salivation. Salivation and regurgitation often cause coughing as patients attempt to swallow and may simulate an esophagorespiratory fistula.

\section{Palliative endoscopic options for dysphagia}

\section{Nasogastric tube}

The esophagoscopic treatment of dysphagia may be achieved by nasogastric tube, dilation, laser treatment, photodynamic treatment, prostheses insertion, or performing a gastrostomy. These different options have specific indications. The purpose of inserting a nasogastric tube is feeding liquid food, and it is an alternative to gastrostomy. The indication is a non-dilatable fibrotic postoperative or postradiotherapy stenosis.

The nasogastric tube presents several disadvantages:

- esthetic - the patient is obliged to live with the tube coming out of his or her nose;

- social - the patient is deprived from a normal social life;

- functional - the external surface of the tube adheres strictly to the inner surface of the stenotic tract and this prevents saliva being swallowed leading to salivation;

- sensuous - with food introduced through the tube, the patient is unable to enjoy its taste, one of the few pleasant sensations remaining at this stage of the life.

These disadvantages have to be considered also when planning to perform a gastrostomy.

\section{Dilation}

Dilation can be performed by pneumatic balloon dilators or by plastic bougies: both can enlarge the esophageal lumen up to $20 \mathrm{~mm}$. The drawback of the dilation is that the stenosis will recur in 1-3 weeks and dilations must be frequently repeated.

\section{Laser treatment}

Laser radiation causes thermal coagulation-destruction of the cancer tissue. The treatment is precise and safe in appropriate hands and the esophageal lumen can be fully restored so to obtain a satisfactory eating function; the mean duration of the patency of the lumen is estimated to be 4-8 weeks.

Photodynamic therapy

Photodynamic therapy (PDT) uses photosensitizing drugs from the group of porphyrins. The photosensitizer, selectively fixed by the tumor and activated by light, produces singlet oxygen that is toxic for biologic tissues and causes a necrotic effect; the necrotic effect of PDT needs 4-8 days to become apparent and the relief from obstruction lasts for 5-10 weeks. Patients submitted to PDT have to avoid direct sunlight for 4-6 weeks because of the skin photosensitization.

\section{Prosthesis}

Inserting a trans-stenotic prosthesis avoids the recurrence of the obstruction due to the regrowth of the tumor. The best results are obtained when the prosthesis does not interfere with the mechanism of a sphincter (the pharyngo-esophageal or the cardiac sphincter). When the cardiac sphincter is infiltrated by the tumor, and the prosthesis keeps it open, the valvular antireflux mechanism is impaired and the gastric content flows back into the esophagus. The acid gastric secretion can be responsible for supra-prosthetic esophagitis and this condition causes dysphagia even though the esophagus is patent. Special prostheses with antireflux mechanisms have been recently manufactured, but definite results of their use are not yet available. When the pharyngoesophageal sphincter is involved and the insertion of the prosthesis keeps it open, the patient must adapt the swallowing mechanism to this new condition and that may need some days or weeks to be perfected. 
The progression of the cancer through the esophageal wall causes often a fistula, connecting the esophageal lumen with the skin of the neck, the trachea, a bronchus, the mediastinum or the pleural space. Prostheses are used to bypass the fistula, allowing immediate passage of the oral intake. Fistulas can be also consequent to previous surgery or radiotherapy. Insertion of a prosthesis to bypass a fistula allows immediate restoration of oral feeding and curing of concomitant dermatitis (in cervical fistulas), bronchopneumonia (in tracheoand broncho-esophageal fistulas), mediastinitis (in mediastinal fistulas) and pleural effusions (in esophago-pleural fistulas). Obviously, only plastic stents or covered mesh stents can be used in the indication of closing a fistulous passage, because a simple mesh stent would allow the filtration of liquids through the mesh. The new self-expanding plastic stents have been used in the treatment of thoracic leaks after esophagectomy for cancer; these stents can be easily removed after the fistula repair and their application reduces leakrelated morbidity and mortality and can be considered as a cost-effective alternative to surgery and to the other endoscopic treatments.

If an esophago-tracheal or an esophago-bronchial fistula cannot be treated by inserting a stent into the esophagus (no concomitant stenosis to avoid migration of the stent), the prosthesis can be inserted into the respiratory tract to close the tracheal or the bronchial opening to avoid aspiration pneumonia, a frequent cause of death in these patients

\section{Percutaneous gastrostomy}

Percutaneous endoscopic gastrostomy (PEG) consists of the insertion of a feeding/venting tube into the stomach, through the abdominal wall under direct endoscopic control. Percutaneous gastrostomy for feeding purposes is considered only when there is no possibility to carry out one of the previously described procedures. A gastrostomy tube can be advanced through the pylorus to reach the jejunum, thus performing a jejunostomy, and it can be used also for decompressing an obstructed intestine.

\section{Bleeding and vomiting}

These symptoms result mainly from intragastric or pancreatic tumors infiltrating the gastric wall. They may be endoscopically treated because they produce symptoms linked to the bleeding or to the food progression (vomiting). This second group of symptoms is generally linked to the compression of the gastric antrum. Malignant ulcerations bleed and produce anemic conditions speeding up the progression toward cachexia.

\section{Palliative endoscopic options for bleeding and vomiting}

Endoscopic laser photocoagulation, argon-plasma coagulation, unipolar or multipolar electrocoagulation, cryotherapy, injection of sclerosing drugs, can be used for cytoreductive as well as for hemostatic purposes. The duration of the effect is limited and the recurrence of the bleeding is a rule. The progression of the infiltration of the gastric wall and the presence of polypoid intragastric masses, mainly in the antrum, obstructs the gastric passage and causes gastric distension, nausea and, finally, vomiting. All these symptoms disappear when the obstruction is relieved after an endoscopic treatment. Duodenal obstructions, as well as the gastric ones, can also be treated by performing a translaparoscopic bypass between the gastric body and the first jejunal loop, although expandable prostheses are also used to bypass duodenal and gastric compressive and stenosing lesions. Duodenum and gastric antrum can be obstructed by primary or metastatic tumors. Malignant lymph nodes, pancreatic and ampullary cancers are the most frequent causes of duodenal obstruction. Together with biliary tumors, these conditions are responsible for biliary obstruction and cause malignant jaundice.

\section{Malignant jaundice}

Pancreatic cancer is the most common cause of malignant biliary obstruction, followed by cholangiocarcinoma, carcinoma of the papilla of Vater, and metastatic tumors. When biliary obstruction and the consequent jaundice occurs, the patient has often advanced stage disease and palliation of the jaundice is the real purpose of the treatment. 


\section{Palliative endoscopic options for malignant jaundice}

Endoscopic stenting after diagnostic transpapillary cholangiography has become the palliative treatment of choice. Palliative treatments alternatives to the endoscopic ones (surgical and percutaneous) have higher costs and complications and a lower success rate. They are indicated in case of failure of the endoscopic procedure.

Insertion of gastroduodenal prostheses can relieve a concomitant obstruction of the gastric outlet and of the gastroduodenal passage. Biliary plastic stents present a main late complication consisting of the occlusion with biliary sludge, generally within 6 months. Metallic expandable prostheses, similar to those used in the coronary vessels, in the urethra and in the tracheobronchial tree, have been proposed for the biliary tract. They have a low occlusion and complication rate, are easy to place and can be considered as a permanent procedure in the malignant jaundice.

The main advantages of the endoscopic approach are the low rate of trauma and the immediate effect. In fact, whitish stools become well-stained in the 24-48 hours after the procedure, the urine loses progressively its intensive brown color and itchiness disappears. When endoscopic drainage is impossible, percutaneous, video-laparoscopic or open surgical routes can be used: the first one allows the insertion of a transhepatic tube, whereas the second and third allow wide exploration of the peritoneal cavity and the performance of a bilio-digestive bypass.

\section{Constipation}

More than $50 \%$ of advanced cancer patients need to be treated for the infrequent passage of hard stool. When caused by anticancer chemotherapeutic drugs (mainly vincristine), opioids, metabolic problems like hypokalemia or global electrolyte imbalances, it is mostly of the type of adynamic ileus and is frequently accompanied by generalized abdominal pain. Intestinal obstruction by endoluminal masses or by extraintestinal compressions is more frequently accompanied by colicky pain and can be endoscopically palliated.

\section{Palliative endoscopic options for constipation}

Endoscopy can help in perfecting the diagnosis as well as in treating the distension. These patients are generally submitted to nasogastric intubation or other venting procedure for gastroduodenal decompression. A transanal approach can be necessary, so that, after cleaning enemas have been performed at low pressure, colonoscopy with large channel endoscopes allows gas and liquid aspiration. Endoscopy must be performed carefully, injecting small quantities of warm water to clean the lumen, considering that the bowel wall is often very fragile because of concomitant ischemic lesions.

\section{Obstruction}

Obstruction can be due to primary cancer, to metastatic involvement of the mesenteric lymph nodes or diffuse peritoneal nodular metastases, e.g. in papillary carcinomas, mainly ovarian and pancreatic. About 10 percent of intestinal obstructions are caused by malignancies; up to 30 percent of obstructions result in resolution. More than 60 percent of malignant obstructions are caused by recurrent cancers.

\section{Palliative options for obstruction}

If a patient, already treated for an intestinal tumor, presents with abdominal distension, vomiting, constipation and crampy pain, and plain X-ray of the abdomen shows air-fluid levels and bowel distension, the first choice is endoscopy. This is to locate precisely the cause of the obstruction and to perform, when possible, the first palliative treatment by dilation, laser, or stenting.

\footnotetext{
Surgery

Surgical procedures can be classified as bowel resections, stomies or bypass operations; the kind of procedure is related to the position of the stenosing lesion and to the type of previous surgery.

Considering the pros and the cons of the different surgical procedures, on the one hand bypass operations and diverting stoma do not remove the tumor and consequently do not interfere with symptoms related to its presence, such as bleeding and pain due to infiltration of anatomic struc-
} 
tures (peritoneum in intra-abdominal cancers or periosteum in the pelvic localizations) although they alleviate symptoms due to obstruction. On the other hand surgical removal, when possible, alleviates all the tumor-related symptoms, but morbidity and mortality rates are higher in patients who undergo palliative versus curative surgery.

Persistence of bowel obstruction has a strong influence on prognostic outcome. Conventional medical treatment has to be established with nasogastric or nasojejunal intubation, and supply of intravenous fluids and electrolytes. The patient must be monitored with serial physical examinations performed by the same physician, enemas should be performed to clean the colon and a colonoscopy should be done with the purpose of localizing and possibly treating the obstructing lesion. This type of examination must be performed carefully by an experienced operator because of the fragility of the patient and of the intestinal wall, of the hypersensitivity to pain, and the risk of perforation.

\section{Endoscopic options}

Dilation, electrocoagulation, laser coagulation, cryotherapy and, lastly, endoprostheses, are the endoscopic procedures to be considered in case of obstruction: all of them have low mortality and morbidity. Any treatment has to be performed only in patients presenting symptoms (obstruction, pain, bleeding) clearly attributable to the tumor and must be directed to bypass the symptom. In asymptomatic patients any palliative treatment must be deferred. These patients generally present with cachexia and weight loss and surgical operations carry a high mortality, about 10 percent, and a survival rate of around 5 percent at 5 years.

In presence of an obstructing mass it has to be removed to reopen the intestinal lumen; this can be done through an endoscopic laser treatment. In case of a lesion infiltrating the bowel wall, dilation has to be performed to reestablish the fecal transit. If the tumor is operable, the lesion can be resected or a bypass operation performed through a surgical laparotomy or a laparoscopic approach in the following days; if the patient is inoperable because of high risk conditions or because the lesion is not removable, the endoscopic alternative is the only feasible option. To keep open the intestinal lumen and maintain the bowel functions, an expandable prosthesis must be inserted. The results of the endoscopic treatments can be summarized as follows:

- dilation with inflatable balloons has a high rate of success ( -90 percent), but stenosis recurs in 1-2 weeks;

- similar rate of success is obtained with Nd:YAG laser but the duration of the dilation is longer;

- insertion of a prosthesis allows the patency of the large bowel to be maintained in more than 80 percent of treated patients.

We started to insert stents in primary rectal tumors and in recurrences in rectal anastomoses with a success rate of more than 90 percent. In most of our cases patency of the stent lasts until the death.

\section{Conclusion}

The features of endoscopic palliation are: (i) achievement of an immediate result in the control of symptoms and in the restoration of a normal function, whereas other options of palliation, like radiotherapy and chemotherapy and surgery, are generally more risky, and need incomparably longer times to become effective; (ii) absence of contraindications and side effects; and (iii) the possible combination of endoscopic treatments with any other form of treatment.

When dealing with an oncologic patient for palliative purposes, one should learn to give the patient the opportunity to fully explain the symptoms of the disease. A combination of an accurate clinical history and the results of the investigations often allows planning of treatment with a reduced number and frequency of traumatic and time-consuming examinations in these patients with a limited survival time.

Palliative treatments, in each case, should be tailored to the individual patient, and some clinical benefit, subject to the patient's capacity to undergo the treatments, consisting of a decrease or disappearance of symptoms and of improvement of performance status, should be the primary endpoint. 


\section{References}

Baines MJ. Symptom control in advanced gastrointestinal cancer. Eur J Gastroenterol Hepatol. 2000, 12: 375-9.

Baron TH, Rey JF, Spinelli P. Expandable metal stent placement for malignant colorectal obstruction. Endoscopy 2002; 34: 823-30.

Ikeda M. Significant host and tumor-related factors for predicting prognosis in patients with esophageal carcinoma. Ann Surg. 2003, 238: 197-202.

Silberman AW. Surgical debulking of tumors. Surg Gynecol Obstet. 1982, 155: 577-85.

Costamagna G. Therapeutic biliary endoscopy. Endoscopy 2000, 32: 209.

Spinelli P, Mancini A, Dal Fante M. Endoscopic treatment of gastrointestinal tumors: indications and results of laser photocoagulation and photodynamic therapy. Semin Surg Oncol. 1995, 11: 307-18.

Spinelli P, Mancini A. Use of self-expanding metal stents for palliation of rectosigmoid cancer. Gastrointest Endosc. 2001, 53: 203-6.

Spinelli P, The endoscopic treetment of gastrointestinal symptoms. In Bruera E, Higginson I, von Gunten C and Ripamonti C Hoddereducation Publisher London, 2006. 